\title{
Asbestiformer Antigorit, ein Risikomineral in Serpentiniten: Argumente für eine Gesundheitsfürsorge entsprechend der Gruppe der Asbestminerale
}

\author{
Walter Vortisch ${ }^{1}$ und Xaver Baur ${ }^{2}$ \\ ${ }^{1}$ Lehrstuhl für Erdölgeologie, Department Angewandte Geowissenschaften, Montanuniversität Leoben, Leoben, \\ Österreich \\ ${ }^{2}$ European Society for Occupational and Environmental Medicine, EOM e.V., Berlin, Deutschland
}

Eingegangen 21. Oktober 2017; angenommen 8. November 2017; online publiziert 30. November 2017

\begin{abstract}
Zusammenfassung: Hohe Asbestfasergehalte der Tunnelluft (bis zu 1,2 $\times 10^{6} / \mathrm{m}^{3}$ [Dissauer et al.]), bei Tunnelvortriebsarbeiten im antigoritischen Serpentinit von Traföß sind zu einem großen Anteil auf die Bildung asbestiformer Antigoritfasern durch die mechanische Bearbeitung des Antigorits zurückzuführen. Der massig erscheinende antigoritische Serpentinit, wie auch gelegentlich beobachtete massige Erscheinungsform chrysotilführender Gesteinsproben, lassen dieses Risiko nicht erkennen. Chrysotil und asbestiformer Antigorit sind mittels Normmethoden (Raster-Elektronenmikroskopie, energiedispersive Spektroskopie) nicht sicher unterscheidbar. Die dem Chrysotil gleichzusetzende, gesundheitsschädigende Wirkung von asbestiformem Antigorit ist im internationalen Schrifttum seit längerem dokumentiert. Asbestiformer Antigorit (sowohl primärer als auch durch mechanische Bearbeitung entstehender) sollte in die Gruppe der Asbestminerale aufgenommen werden, mit entsprechenden rechtlichen und arbeitsmedizinisch-präventiven Konsequenzen. Bei Tunnelvortriebsarbeiten in Serpentiniten sollte von Anfang an die Umgebungsluft im Tunnel bezüglich ihres Fasergehaltes kontrolliert werden, um nötigenfalls sofort Arbeits-
\end{abstract}

Zu demselben Thema erscheint ein Artikel der beiden Autoren im Neuen Jahrbuch für Mineralogie [Band 195 (2018), Heft 1, Online 16. November 2017, https://doi.org/10.1127/njma/2017/0070] in englischer Sprache. Wir danken dem Verlag Schweizerbart für die freundliche Genehmigung, die vorliegende komprimierte Fassung (mit Ergänzungen) in deutscher Sprache in den Berg- und Hüttenmännischen Monatsheften veröffentlichen zu dürfen.

Em. Prof. Dr. W. Vortisch ( $\square)$

Lehrstuhl für Erdölgeologie, Department Angewandte

Geowissenschaften,

Montanuniversität Leoben,

Franz-Josef-Str. 18,

8700 Leoben, Österreich

Walter.Vortisch@unileoben.ac.at schutzmaßnahmen, wie sie zum Beispiel von Dissauer et al. beschrieben wurden, veranlassen zu können.

Schlüsselwörter: Serpentinit, Asbestiformer Antigorit, Tunnelbau, Röntgendiffraktometrie, Raster-

Elektronenmikroskopie

Asbestiform Antigorite, a Risk Mineral of Serpentinites: Arguments for Consideration as Asbest Mineral Group Member Concerning Occupational Health Care

Abstract: Exceptionally high contents of asbestos fibres were measured in the tunnel air (up to $1.2 \times 10^{6} / \mathrm{m}^{3}$, Dissauer et al.), during tunnelling works in the antigoritic Traföß serpentinite (Kirchdorf tunnel, S35, Styria). Main source of these fibres is antigorite, forming silicate fibres (asbestiform antigorite) similar to the ones of chrysotile when mechanically worked in the course of the tunnelling process. Due to its massive appearance in hand piece and outcrop the analysed antigorite does not reveal this risk. (Minor occurrences of chrysotile-containing parts of the serpentinite showed this massive appearance as well.) Chrysotile and asbestiform antigorite cannot be distinguished definitely by standard procedures (scanning electron microscopy combined with energy-dispersive spectroscopy). The adverse health effects of asbestiform antigorite, corresponding to the ones of chrysotile, are documented in the international literature. Therefore, asbestiform antigorite, both primarily fibrous as well as secondarily formed by mechanical disruption, should be added to the group of asbestos minerals with corresponding legal consequences for occupational health care. During tunnelling in serpentinites monitoring of the fibre counts in the ambient air should start at the very beginning in order to allow immediate implementation of necessary safety measures (as described e. g. by Dissauer et al.). 
Keywords: Serpentinite, Asbestiform antigorite, Tunnelling, X-ray diffractometry, Scanning electron microscopy

\section{Einleitung}

Beim Bau des Kirchdorf-Tunnels (Kirchdorf/Steiermark, Schnellstraße S35) wurde ein Serpentinitkörper (der Serpentinit von Traföß) auf circa $500 \mathrm{~m}$ durchörtert. Im Tunnelabschnitt des Serpentinits wurden in der Tunnelluft hohe Gehalte an asbestiformen, als Chrysotil interpretierten Silikatfasern festgestellt (bis zu $1,2 \times 10^{6} / \mathrm{m}^{3}$, Dissauer et al. [1]). Bei früheren Untersuchungen [2] sowie im Rahmen der Vorerkundung der Tunneltrasse [1] war im Gegensatz zu den Problemen im Tunnelvortrieb nur der im Allgemeinen als blättchenförmig bezeichnete Antigorit im Serpentinit von Traföß beobachtet worden. Chrysotil, das typische Asbestmineral der Serpentinmineralgruppe (Antigorit, Lizardit, Chrysotil), war im Rahmen der früheren Untersuchungen nicht festgestellt worden. Auch im Bescheid des Amtes der Steiermärkischen Landesregierung (2007) wird bezüglich des Mineralbestandes der Fraktion $<2 \mu \mathrm{m}$ Antigorit als dominantes Mineral genannt. Die anderen Serpentinminerale (Chrysotil, Lizardit) werden nicht erwähnt. Geringere Vorkommen von Chrysotil waren nur von dem stratigraphisch und lithologisch dem gleichen Kristallingesteinskomplex (dem altpaläozoischen SpeikKomplex) zugehörigen Serpentinit von Kraubath bekannt [3]. Antigorit ist ein dominantes Mineral der im Alpenraum verbreiteten Serpentinite.

Umfangreiche Untersuchungen von Proben aus den dem Kirchdorf-Tunnel benachbarten Aufschlüssen (Weganschnitte oberhalb der südlichen Tunnelportale) und des Tunnelausbruchsmaterials bestätigten den im Wesentlichen antigoritischen Charakter des vom Kirchdorf-Tunnel durchörterten Serpentinits. Untergeordnet konnte auch Chrysotil nachgewiesen werden. Eine Ausnahme stellte eine chrysotilreiche Aufschlussprobe dar (Chrysotilgehalt nahe $90 \%$ ), die einer schmalen, überwiegend hangschuttbedeckten vermutlichen Bruchzone entnommen wurde (nähere Einzelheiten zu den mineralogischen Untersuchungen siehe Vortisch und Baur [4]). Entsprechend den hohen Silikatfasergehalten der Tunnelluft wurden auch zahlreiche asbestiforme Fasern in Proben des Feinanteils des Tunnelausbruchsmaterials beobachtet. Es stellte sich daher die Frage nach der Herkunft der asbestiformen Silikatfasern, beziehungsweise ob der im Serpentinit von Traföß auftretende Antigorit die wesentliche Quelle der beobachteten asbestiformen Silikatfasern sein kann. Zu berücksichtigen ist in diesem Zusammenhang, dass auch für Antigorit faseriger, asbestiformer Habitus mit entsprechenden Risiken für die menschliche Gesundheit seit längerem bekannt ist, worauf im Folgenden näher eingegangen wird.

\section{Faseriger Antigorit}

In umfassenden Studien zeigen Fitz Gerald et al. [5], Groppo und Compagnoni [6] und Keeling et al. [7-9], dass Antigorit in ähnlicher faseriger Form auftreten kann wie Chrysotil (Faserdicke $<1-5 \mu \mathrm{m}$, Faserlänge bis mehrere $100 \mu \mathrm{m}$, siehe z. B. rasterelektronenmikroskopische Aufnahmen von Keeling et al. [7]). In ihren rasterelektronenmikroskopischen Aufnahmen zeigen letztere Autoren alle Übergänge von länglich-leistenförmigen bis feinfaserigen Erscheinungsformen. Offensichtlich kann leistenförmiger, d.h. noch eher länglich-plattig erscheinender Antigorit leicht in die typischen feinen asbestiformen Fasern zerlegt werden.

Bezüglich der kristallographischen Ursache für die Faserbildung unterscheiden sich Chrysotil und Antigorit. Bei Chrysotil sind die Kristallite zu feinen Röhrchen („tubes“) aufgerollt, während bei Antigorit verringerte Kohäsion zwischen (Kristall-)Gitterdomänen die Bildung leistenförmiger Kristallite, mit häufigen Durchmessern quer zur Längserstreckung im 1er $\mu \mathrm{m}$ - Bereich und Längen im 10er bis $100 \mathrm{er} \mu \mathrm{m}$-Bereich, verursacht und Gitterdefekte aufgrund chemischer Variation zur weiteren Zerlegung zu feineren Fasern führen, bzw. deren Zerlegung zu solch feineren Fasern entsprechend fördern. Bei den feinen Fasern können Durchmesser quer zur Längserstreckung bis zu $<0,2 \mu \mathrm{m}$ auftreten [5]. Ein mechanischer Unterschied besteht nach den genannten Autoren darin, dass Chrysotilfasern häufig elastischer sind als Antigoritfasern.

Das bedeutende Gesundheitsrisiko von faserigem Antigorit wurde bereits von einer Reihe von Veröffentlichungen behandelt (Keeling et al. [9]; Baumann [10]; Cardile et al. [11]; Pugnaloni et al. [12]; Wozniak et al. [13, 14]).

Bezüglich der Definition von Silikatfasern wird im Weiteren der ÖNORM M9405 (Messung von Asbestfaserkonzentrationen in der Luft [15]) gefolgt, die festlegt: "Als anorganische Faser ist jedes Objekt zu bestimmen, das eine Länge von $5 \mu \mathrm{m} \leq L \leq 100 \mu \mathrm{m}$, einen Durchmesser $D<3 \mu \mathrm{m}$, ein Länge/Durchmesser-Verhältnis $L: D \geq 3: 1$ sowie weitgehend parallele Kanten in Längsrichtung aufweist und bei der EDX-Analyse ein Elementspektrum liefert." Wobei zu berücksichtigen ist, dass auch anders dimensionierte, zum Beispiel kürzere asbestiforme Silikatfasern ein bedeutendes Gesundheitsrisiko darstellen können [16-18].

Wichtig ist, dass mittels normengerechter Analyse (ÖNORM M 9405, 1993 [15]; VDI 3492, 2013 [19], RasterElektronenmikroskopie kombiniert mit energiedispersiver Spektroskopie, REM/EDS) Chrysotil und asbestiformer Antigorit nicht sicher unterschieden werden können, da beide chemisch und morphologisch sehr ähnlich sind (vgl. auch Abb. 5-10).

Auch die röntgendiffraktometrische Unterscheidung der sowohl chemisch wie auch kristallographisch sehr ähnlichen drei Mineralspezies der Serpentinmineralgruppe (Antigorit, Lizardit, Chrysotil) ist schwierig. Die Bestimmung des Antigorits, vor allem wenn er als Hauptgemengtheit auftritt, ist hingegen anhand der Daten von Keeling et al. [9] auch mittels Röntgendiffraktometrie unproblematisch. Eine Unterscheidung von Chrysotil und Lizardit, wenn sie als Nebengemengteile neben dominantem Antigorit auftreten, ist jedoch schwierig. Dies liegt an der kristallographischen Vielfalt der Serpentinminerale und damit auch der veröffentlichten d-Werte ihrer Röntgenreflexe (z. B. Bailey [20], Wicks und O'Hanley [21]). Dódony und Busek [22] gehen sogar von Übergängen zwischen den einzelnen Serpentin- 


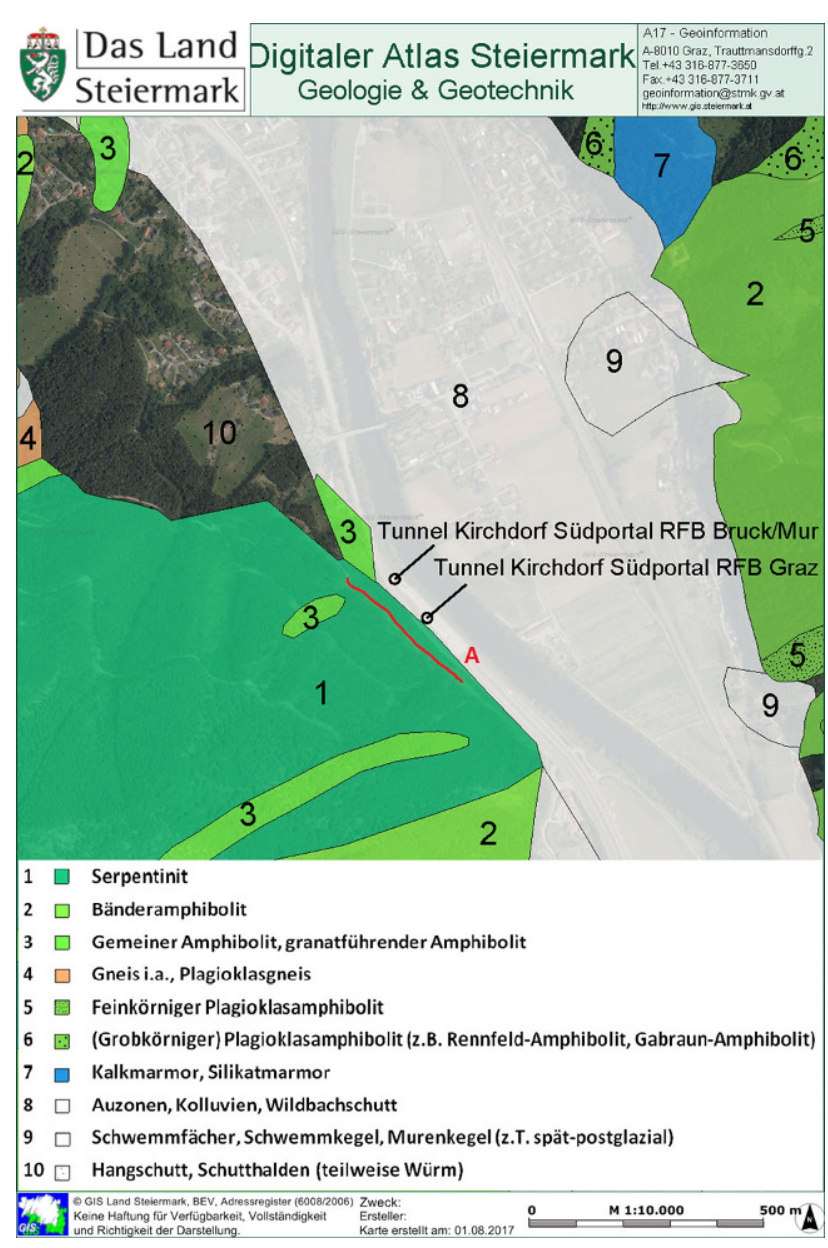

Abb. 1: Lokale Geologie in der Umgebung des Serpentinits von Traföß (Ausschnitt aus dem Digitalen Atlas der Steiermark, Maßstableiste $500 \mathrm{~m}$ ). Die lithologischen Einheiten entsprechen im Wesentlichen jenen von Neubauers Karte des Serpentinit-Amphibolitkomplexes von Traföß [2]. AAbschnitt des Forstweges, in dem Serpentinit und andere Einheiten des Speik-Komplexes aufgeschlossen sind

mineralen aus, was deren klare Trennung voneinander sehr schwierig gestaltet (Weiteres zur Analytik der Serpentinminerale siehe Vortisch und Baur, im Druck [4]). Für die eigenen Untersuchungen konnte die chrysotilreiche Probe 19 (Chrysotilgehalt nahe $90 \%$ ) als Standard für den röntgendiffraktometrischen Vergleich mit anderen Proben verwendet werden. Das Röntgendiffraktogramm dieser Probe entspricht jenem der Probe CLU1 in Abb. 2 von Keeling et al. [9]. Ebenso konnten verhältnismäßig reine Antigoritproben für Vergleichszwecke eingesetzt werden (Probe 4 und Probe SN2.2, siehe Abb. 4)

\section{Geologie des Serpentinits von Traföß}

Der Serpentinit von Traföß ist, wie erwähnt, Teil des altpaläozoischen Speik-Komplexes, ein lithologisch vielgestaltiger polymetamorpher ophiolithischer Kristallingesteinskomplex, der unter anderem auch im Gebiet von Kraubath auftritt $[2,3]$. Wichtige lithologische Einheiten dieses Komplexes wurden von Neubauer beschrieben [2]

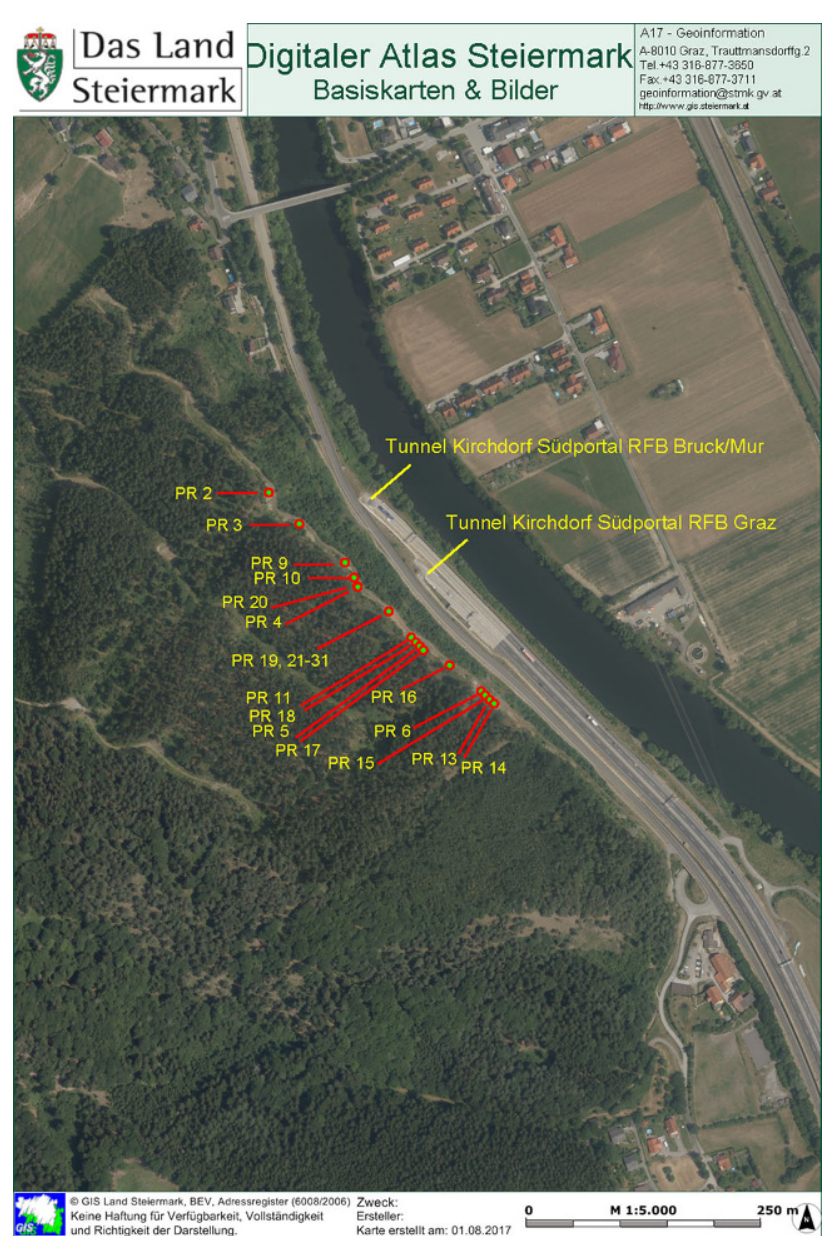

Abb. 2: Probenpositionen im Aufschluss oberhalb der südlichen Tunnelportale (Proben 2-31); Ausschnitt aus dem Digitalen Atlas der Steiermark

und sind in Abb. 1 wiedergegeben. So wie im Rahmen der Voruntersuchung der Tunneltrasse wurde auch von Neubauer im Serpentinit von Traföß nur Antigorit beobachtet [2]. Geringe Vorkommen von Chrysotil waren, wie erwähnt, nur vom Serpentinit von Kraubath bekannt [3]. Allerdings wurde später von den Mineraliensammlern Polenschak und Gesselbauer Chrysotil in einer Halde von Tunnelausbruchsmaterial des Kirchdorf-Tunnels beobachtet (Niedermayr et al. [23, S. 266]).

\section{Eigene Untersuchungen}

\subsection{Probennahme und Untersuchungsmethoden}

Aus dem Aufschlussbereich oberhalb des Tunnelportals wurden insgesamt 28 Proben des Serpentinits von Traföß und begleitender anderer Kristallingesteine des SpeikKomplexes entnommen (Proben 2-31, Abb. 2; Probe 1: gröBerer Hangschuttblock $300 \mathrm{~m}$ NW der Aufschlussprobe 2). Weiters wurden aus 4 Baggerschürfen (SN2-SN5) in der 


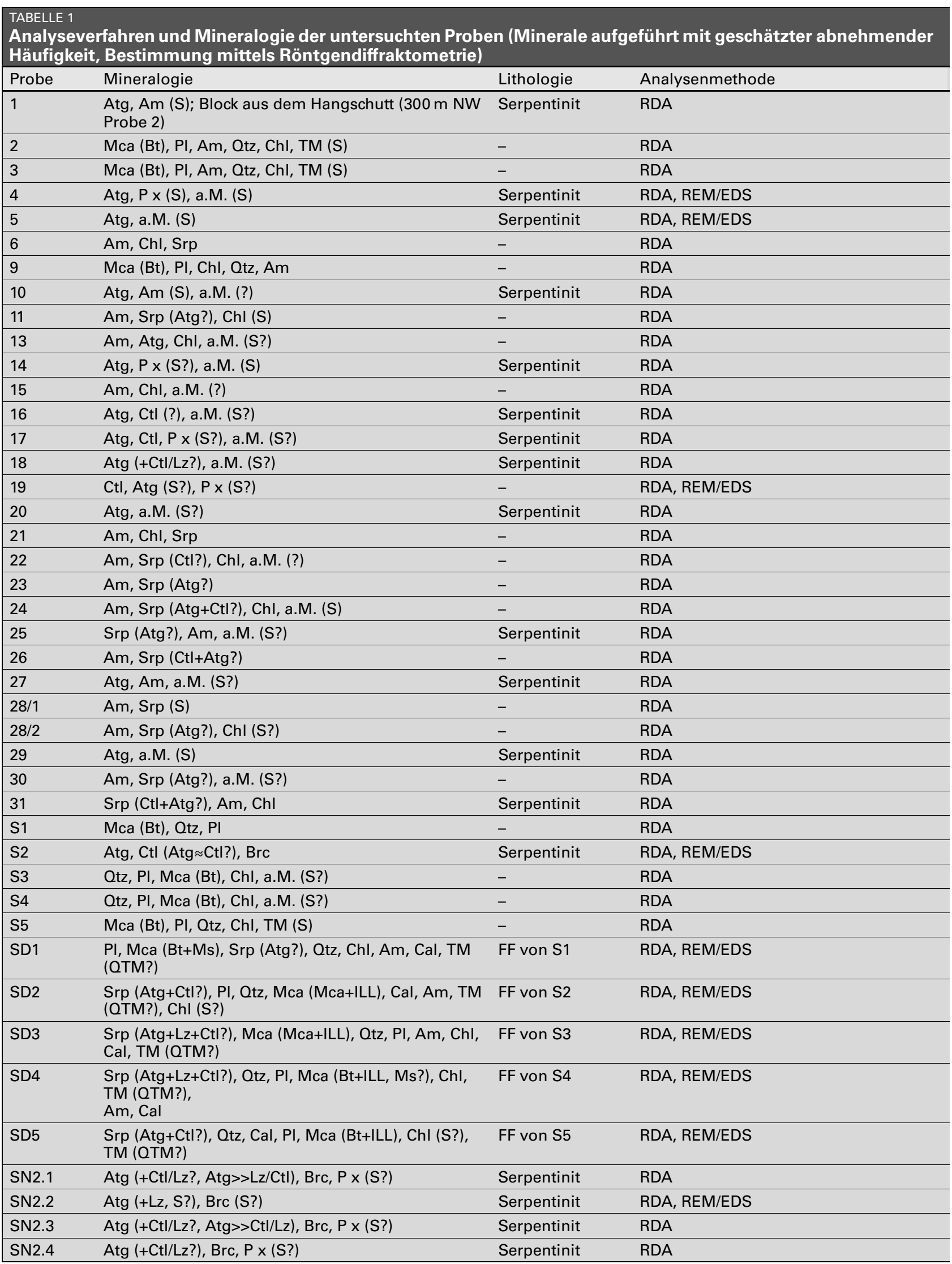




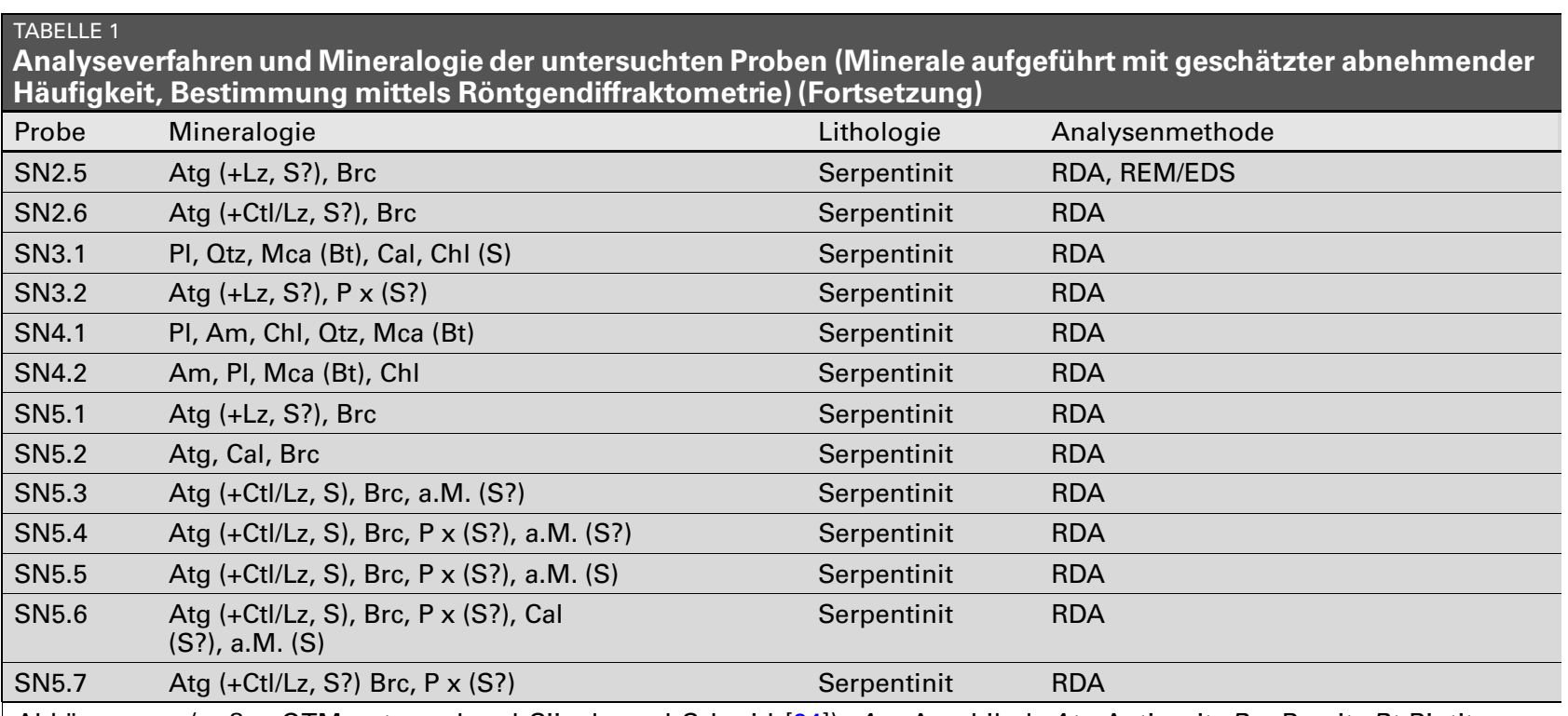

Abkürzungen (außer QTM entsprechend Siivola und Schmid [24]): Am Amphibol, Atg Antigorit, Brc Brucit, Bt Biotit, Cal Calcit, Chl Chlorit, Ctl Chrysotil, FF Feinfraktion (feinkörniges Material entnommen von der Oberfläche der Gesteinsfragmente), ILL Illit, Lz Lizardit, Mca Glimmer, Ms Muscovit, PI Plagioklas, OTM quellfähige Tonminerale, Otz Quarz, Srp Serpentinmineral, TM Tonminerale, S Spuren, a.M. andere Minerale (nicht identifiziert). Untersuchungsmethoden: $R D A$ röntgendiffraktometrische Analyse, REM/EDS Raster-Elektronenmikroskopie mit energiedispersiver Spektroskopie. Zusätzlich wurden 9 Proben von Feinfraktionen der Baggerschürfe SN2, SN3, SN4 und SN5 mittels REM/EDS analysiert (Proben SN2a, SN2c, SN2d, SN3b, SN5a, SN5c: feinkörniges Material auf Gesteinsbruchstücken; Proben SN3a, SN4a, SN5ges: gemischtes feinkörniges Material aus dem Baggerschurf).

Abb. 3: Handstücke der praktisch rein antigoritischen Proben 4 (Aufschluss) und SN2.2 (BaggerschurfSN2) sowie der Aufschlussproben 19 (nahe $90 \%$ Chrysotil) und 17 (Antigorit und Chrysotil in ähnlichen Größenordnungen; Länge der Hammerspitze: ca. $7 \mathrm{~cm}$ Pfeile Beprobte Teilproben fürRöntgendiffraktometrie) Röntgendiffraktogramme dieser Proben sind in Abb. 4 wiedergegeben. Die Proben 4 und SN2.2 wurden für den mechanischen Test verwendet (siehe Abb. 7 und 8); rasterelektronenmikroskopische Aufnahme der Probe 19 siehe Abb. 9

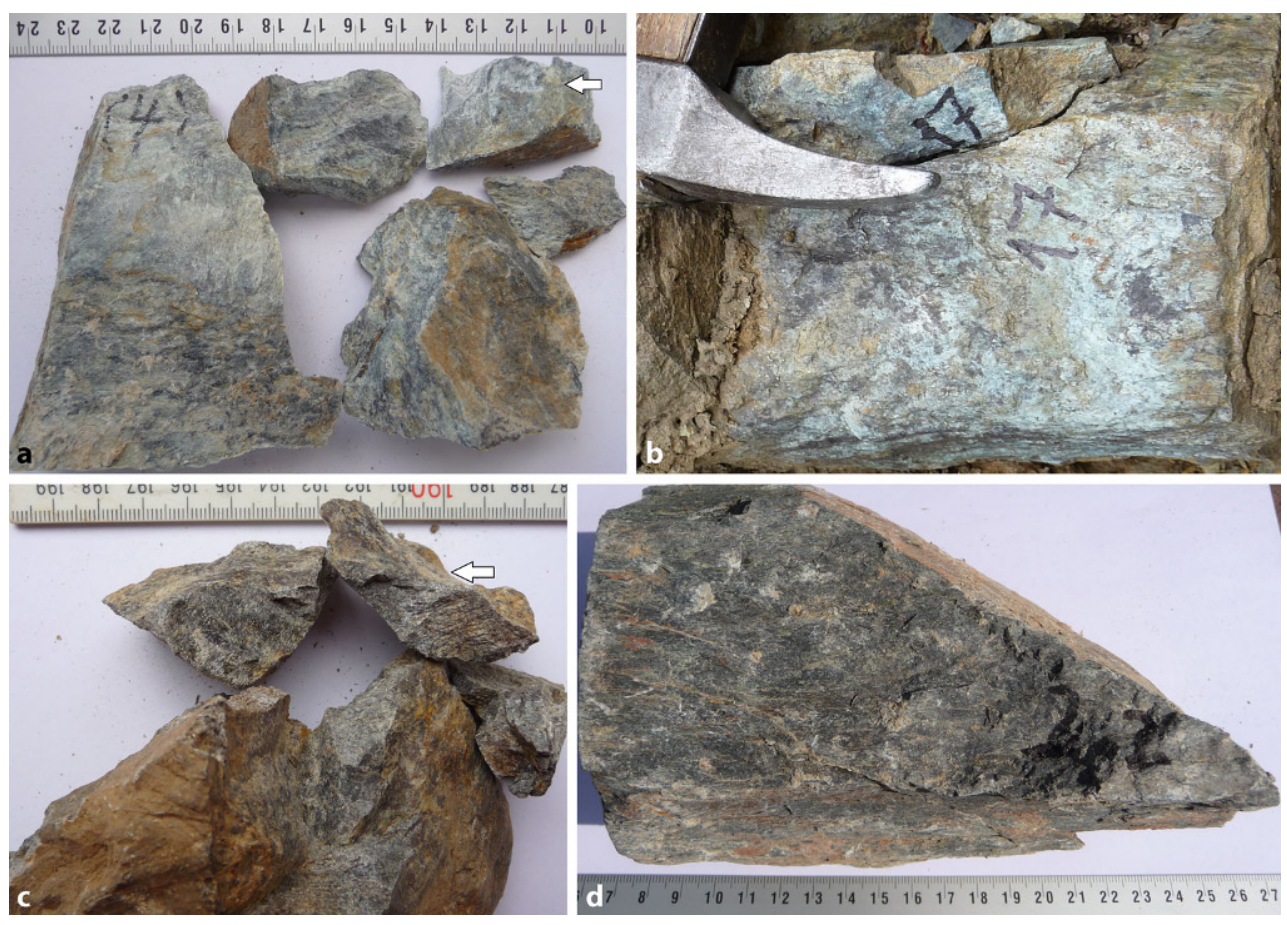

Deponie des Tunnelausbruchsmaterials 17 Proben entnommen. Sämtliche Proben wurden durch den erstgenannten Autor entnommen. Zusätzlich standen Proben aus 5 früheren Baggerschürfen zur Verfügung (je ein Handstück/ Baggerschurf, S1-S5). Des Weiteren wurde Feinmaterial aus den Baggerschürfen, überwiegend anhaftend an Ge- steinsbruchstücken, entnommen und ebenfalls analysiert (insgesamt 14 Proben; Schürfe S1-S5: Proben SD1-SD5; Schurf SN2: SN2a, SN2c, SN2d; Schurf SN3: SN3a, SN3b; Schurf SN4: SN4a; Schurf SN5: SN5a, SN5c, SN5ges; Übersicht über alle Proben siehe Tab. 1). 


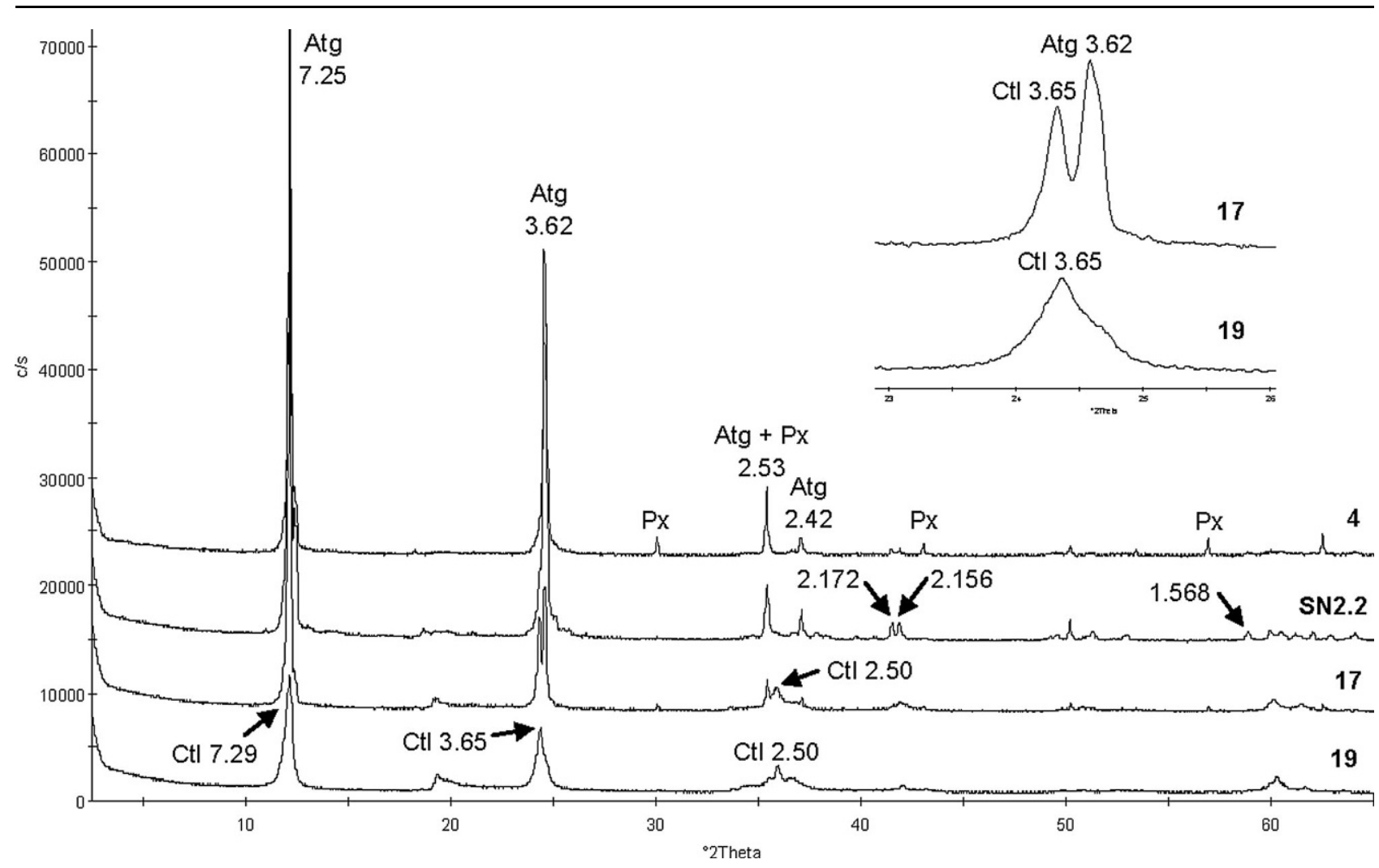

Abb. 4: Röntgendiffraktogramme der praktisch rein antigoritischen Proben 4 und SN2.2 sowie der chrysotilreichen Probe 19 und der chrysotilführenden Probe 17; Strahlung $\mathrm{CuK}_{\alpha 1}, \mathrm{CuK}_{\alpha 2}$ rechnerisch eliminiert, d-Werte in Ångström (Handstücke der Proben siehe Abb. 3). In der Probe 17 tritt neben Antigorit in ähnlicher Menge auch Chrysotil auf. Die angegebenen d-Werte der Proben 4, 19 und SN2.2 wurden durch zusätzliche Messungen mit beigemischtem Quarz (als internem Standard) kalibriert. Im Diffraktogramm der Probe SN2.2 sind weitere diagnostische Reflexe des Antigorits gekennzeichnet. Rechts oben: Ausschnitt der Röntgendiffraktogramme der Proben 17 und 19 (23-26 ${ }^{\circ} 2$ Theta). Die antigoritischen Serpentinitproben 4 und SN2.2 wurden für den mechanischen Test (Faserbildung bei Zerkleinerung?) verwendet. Atg Antigorit, Ct/Chrysotil, Px Pyroxen (Spuren)

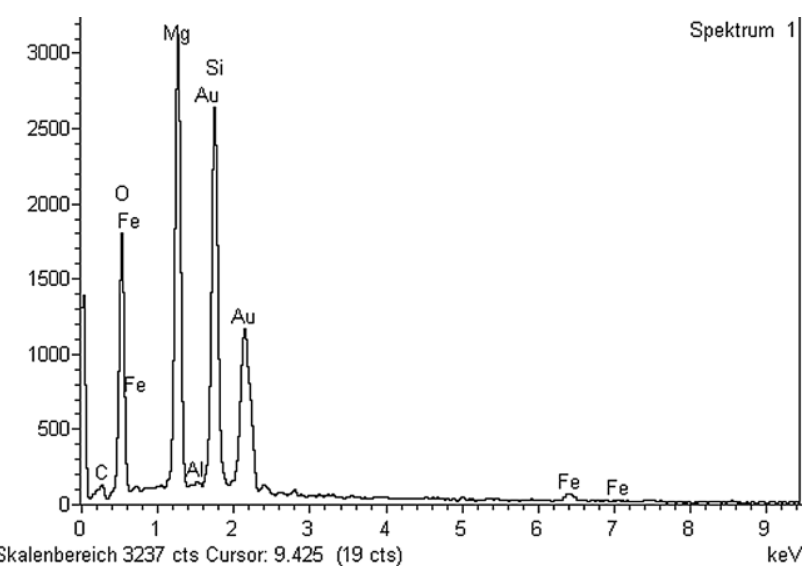

Abb. 5: EDS-Spektrum eines Antigoritfragments der Probe 4 (siehe Abb. 7); Beschleunigungsspannung 20 kV, Goldbedampfung. Das Spektrum zeigt ein Magnesiumsilikat mit niedrigen Gehalten von Eisen und Aluminium. Anmerkung: Chrysotil kann ein identisches EDS-Spektrum liefern (vgl. Abb. 6)

Repräsentative Teilproben der entnommenen Proben wurden nach entsprechender Zerkleinerung röntgendiffraktometrisch analysiert (Pulverpräparate, CuK ${ }_{\alpha}-$ Strahlung, PANalytical X'Pert, Winkelbereich 2-65 ${ }^{\circ} 2 \theta$ ). Eine Auswahl der Proben wurde außerdem rasterelektronenmikroskopisch (mit energiedispersiver chemischer Analytik,
REM/EDS) untersucht (die Feinanteile der Proben aus den Schürfen SN2-SN5 nur mittels REM/EDS; siehe Tab. 1).

\subsection{Untersuchungsergebnisse}

Die Ergebnisse der röntgendiffraktometrischen Analysen sind in Tab. 1 zusammengefasst. Sie zeigen die lithologische Vielgestaltigkeit der im Aufschluss beprobten Gesteine des Speik-Komplexes. Von den insgesamt 50 analysierten Festgesteinsproben repräsentieren 28 Proben Serpentinit. Mit zwei Ausnahmen ist in den Serpentinitproben Antigorit stets das dominante Serpentinmineral. Die weiteren Serpentinminerale (Lizardit, Chrysotil) treten in den Serpentinitproben nur mit geringen, röntgendiffraktometrisch nicht sicher bestimmbaren, Anteilen auf. In einer Schurfprobe (S2) und einer Aufschlussprobe (17) tritt Chrysotil in etwa gleicher Größenordnung auf wie Antigorit. Als nahezu reine Chrysotilprobe erwies sich Probe 19 (siehe Abb. 3 und 4), die aus einer überwiegend mit Hangschutt bedeckten vermutlichen Bruchzone stammt. Die in der Nachbarschaft der Probe 19 entnommenen weiteren Proben (Probe 21-31; Abstand zu Probe 19 soweit anstehend $\leq 1,5 \mathrm{~m}$ ) weisen überwiegend amphibolitischen Charakter auf (Tab. 1). Die chrysotilreiche Probe 19 entspricht daher einer relativ schmalen Zone. Auch bei der chrysotilführenden Aufschlussprobe 17 handelt es sich wahrscheinlich um eine schmale Bruchzone. 


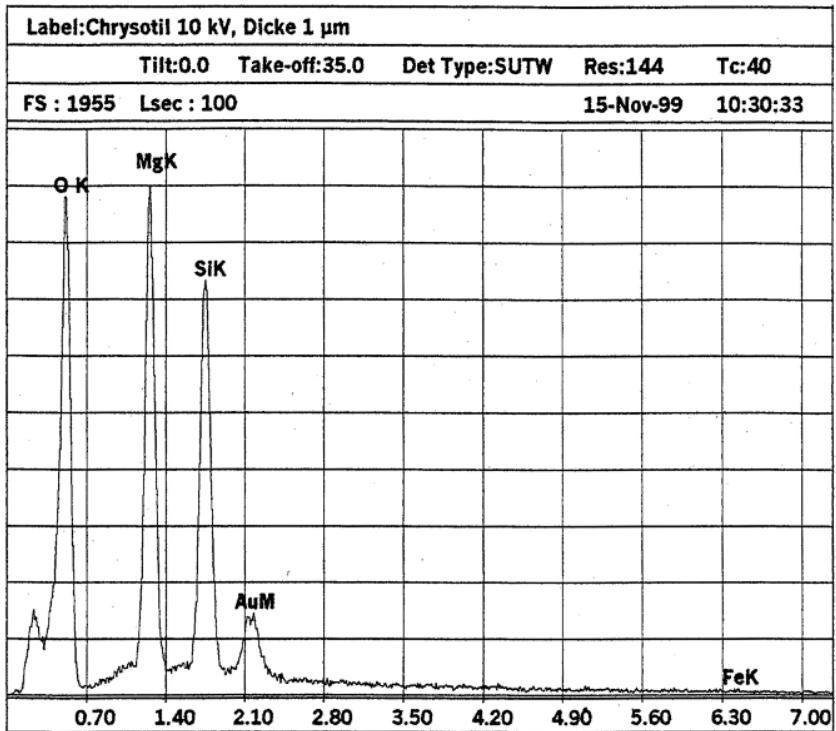

a

Abb. 6: Für den Vergleich mit dem EDS-Spektrum in Abb. 5 (Antigorit): Beispiele von EDS-Spektren (Goldbedampfung) von Chrysotil in Abhängigkeit von der Beschleunigungsspannung; a 10 kV, b 20 kV (VDI Richtlinie 3492, Juni 2013 [19]). Das mit 20 kV erhaltene EDS-Spektrum ist nahezu identisch mit jenen der Abb. 5 (dickere Goldbedampfung im VDI Beispiel). Wiedergegeben mit Erlaubnis des Vereins Deutscher Ingenieure e. V. (VDI)

Diese Probe wurde nur einen Meter südlich der rein antigoritischen Probe 5 entnommen. Bemerkenswerter Weise erscheinen sowohl die chrysotilreiche Probe 19, als auch Probe 17 als Handstück bzw. im Aufschluss unauffällig massig (siehe Abb. 3).

Die praktisch rein antigoritischen Proben 4 (Aufschluss) und SN2.2 (Baggerschurf) wurden für Testzwecke vorsichtig zerkleinert, um die mechanische Belastung während des Tunnelvortriebs zu simulieren. Es zeigte sich, dass sich durch die mechanische Bearbeitung intensiv (oft auffällig elastische) asbestiforme Fasern bilden, die im Gegensatz zu anderen Beobachtungen (z. B. Keeling et al. [7]) auch hinsichtlich ihrer Elastizität nicht von Chrysotilfasern mittels Raster-Elektronenmikroskopie eindeutig unterscheidbar sind (Abb. 7 und 8). Im Gegensatz zur massigen Erscheinungsform zeigte die chrysotilreiche Probe 19 im RasterElektronenmikroskop die für dieses Mineral typische primäre Faserigkeit (Abb. 9).

In allen röntgendiffraktometrisch untersuchten Proben des Feinmaterials der Schürfe S1-S5 (Proben SD1-SD5) bildet Antigorit ein Hauptgemengteil. Daneben sind die weiteren Serpentinminerale (Lizardit, Chrysotil) als Gruppe ebenfalls erkennbar, aber aufgrund der sehr komplexen Gesamtmineralogie der Proben nicht näher differenzierbar. Die rasterelektronenmikroskopische Analyse zeigte in den meisten Proben der Feinanteile aus den Baggerschürfen (Ausnahme SN4) zahlreiche asbestiforme, den Serpentinmineralen zuzuordnende Silikatfasern (Abb. 10).

\section{Diskussion}

Die bei den Tunnelvortriebsarbeiten im Serpentinit von Traföß festgestellten, extrem hohen Asbestfasergehalte der Tunnelluft (bis 1,2 Mio. Fasern $/ \mathrm{m}^{3}$ Luft) sind angesichts
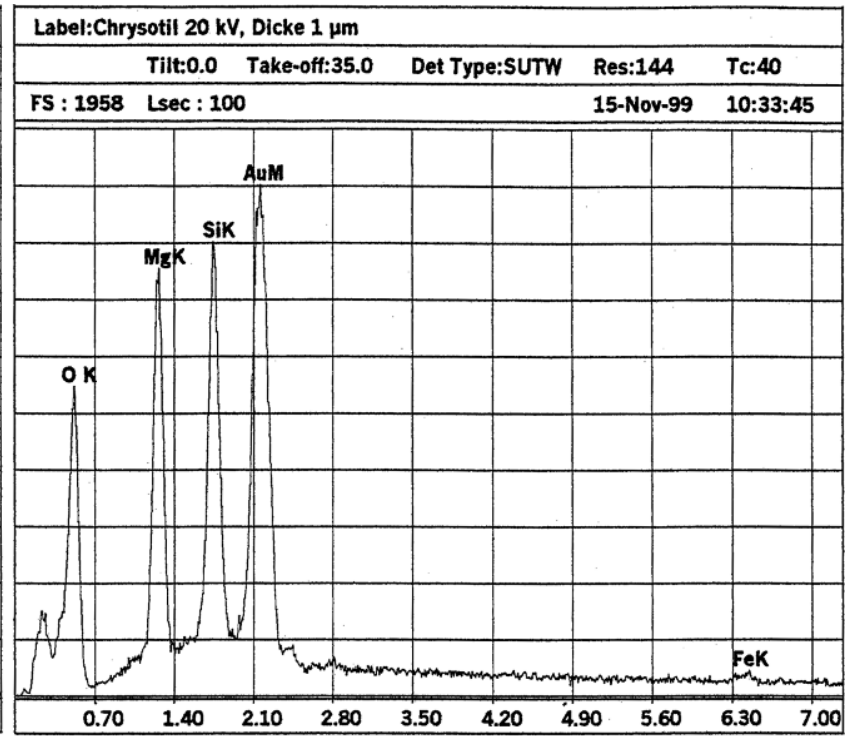

des antigoritischen Charakters dieses Gesteins nur erklärbar, wenn es sich hier um einen Antigorittyp handelt, der bei mechanischer Bearbeitung zur massenhaften Bildung von Silikatfasern führt. Durch die experimentelle Untersuchung (rasterelektronenmikroskopische Analyse von zerkleinertem Probenmaterial, siehe oben) wurde diese Annahme bestätigt.

Die bereits zitierte wissenschaftliche Literatur belegt, dass Antigorit sowohl mit blätterigem als auch mit faserigem Habitus (asbestiform) auftreten kann. Antigorit wird auch im Handbook of Mineralogy (Mineral Data Publishing [25]) als "commonly bladed or fibrous" beschrieben (http:// www.handbookofmineralogy.com/pdfs/antigorite.pdf).

Auch die geringen Eisen- und Aluminiumgehalte passen zum Analysenergebnis, da, wie in Chrysotil, auch in Antigorit substitutionsbedingt geringe Mengen von Aluminium und Eisen auftreten können (vgl. Newman und Brown [26]).

Für die gegebene Fragestellung ist somit wesentlich, dass sich der hier untersuchte Antigorittyp schon bei geringer mechanischer Belastung in feine Fasern auflöst, die, dem internationalen Schrifttum entsprechend, als asbestiform bezeichnet werden. Die massiven Probleme für die Arbeitsplatzsicherheit beim Tunnelvortrieb des KirchdorfTunnels beweisen somit, dass nicht nur Asbestminerale im bisherigen mineralogisch-nomenklatorischen Sinne ein erhebliches Risiko bei Arbeiten in Serpentiniten darstellen können. Aufgrund der morphologischen und kristallchemischen Übereinstimmung mit Chrysotil sind die asbestiformen Fasern des hier auftretenden Antigorits in ihrer gesundheitsgefährdenden Wirkung dem Chrysotil gleichzusetzen (Weiteres siehe Abschn. 5.1).

Im geologischen Handstück bzw. Geländeaufschluss erscheint dieser antigoritische Serpentinit im Allgemeinen 

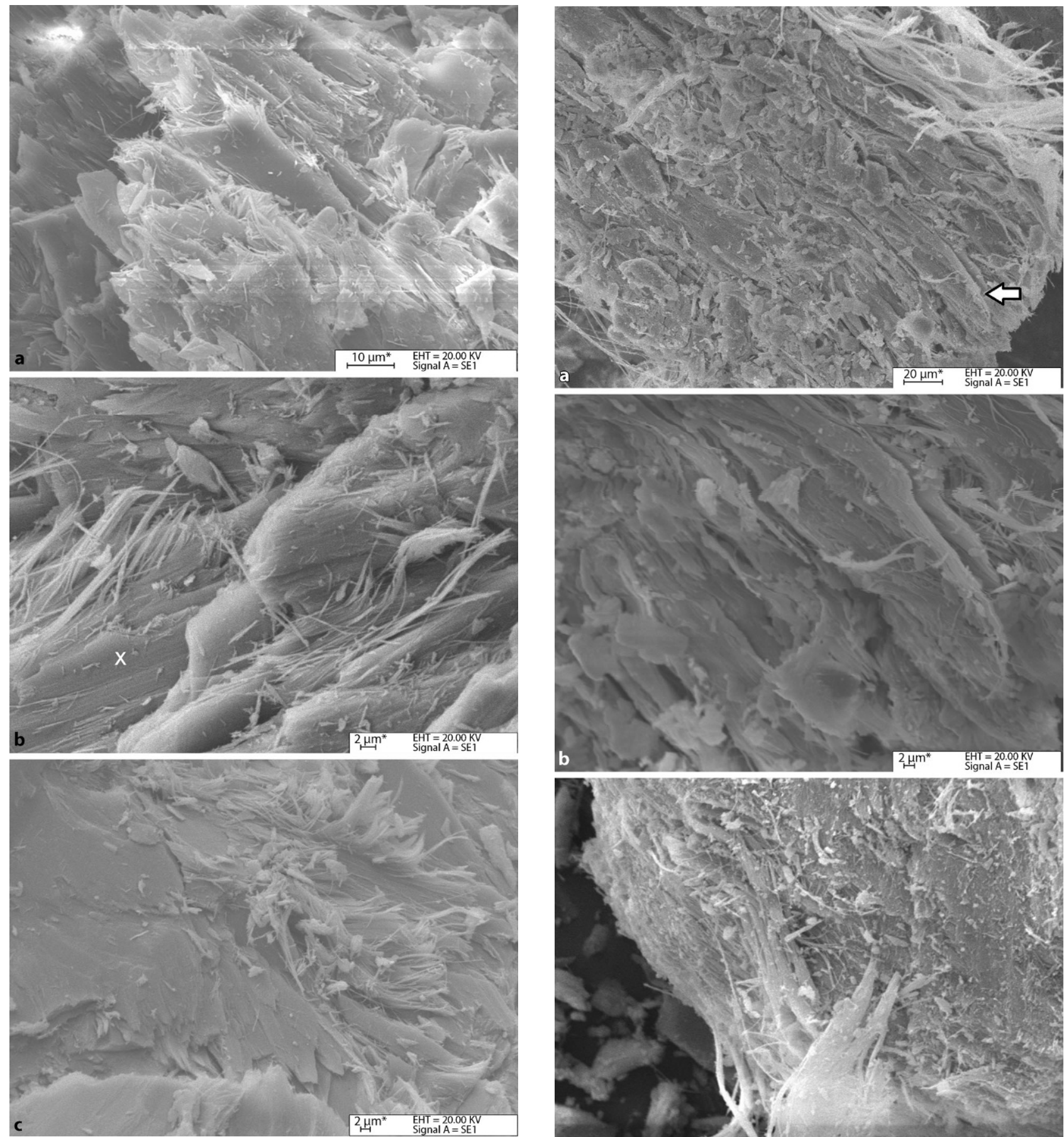

Abb. 7: Rasterelektronenmikroskopische Aufnahmen von Fragmenten der Probe 4 (Aufschluss) nach vorsichtiger Zerkleinerung (Handstücksiehe Abb. 3, Röntgendiffraktogramm in Abb. 4). Es treten alle Übergänge von blättchenförmig zu feinfaserig (asbestiform) auf. XMesspunkt (b) des in Abb. 5 wiedergegebenen EDS-Spektrums
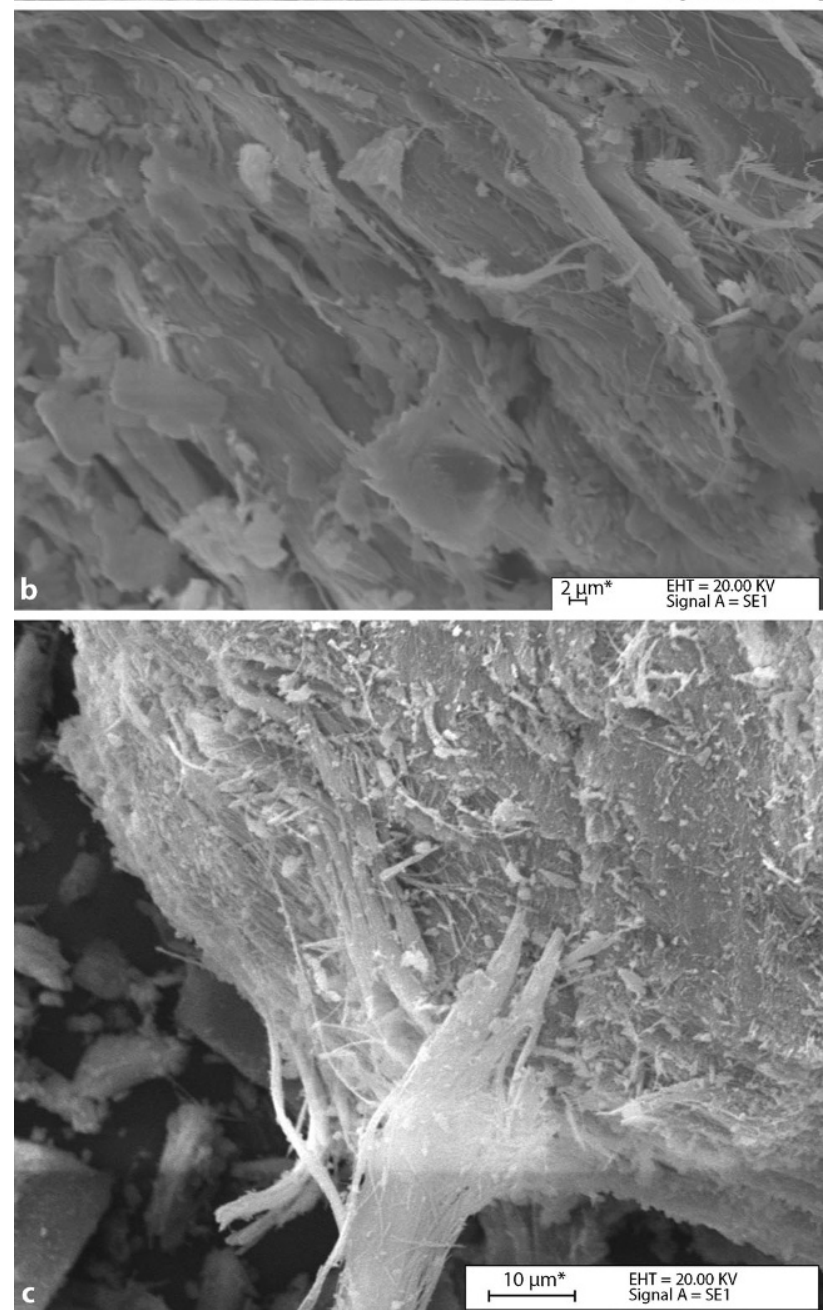

Abb. 8: Rasterelektronenmikroskopische Aufnahmen von Fragmenten der Probe SN2.2 (Baggerschurf) nach vorsichtiger Zerkleinerung (Handstück siehe Abb. 3, Röntgendiffraktogramm in Abb. 4). Es treten, wie bei Probe 4, Übergänge von blättchenförmig zu feinfaserig (asbestiform) auf. b Ausschnitt von a, Position in a gekennzeichnet mit Pfeil 


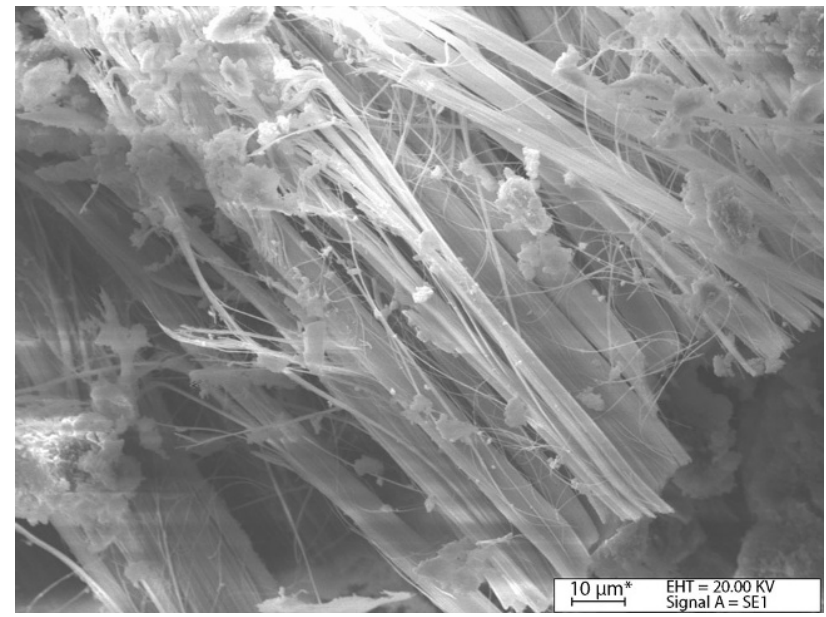

Abb. 9: Für den Vergleich mit Abb. 7 und 8, rasterelektronenmikroskopische Aufnahme eines Fragments der Probe 19 (Chrysotil, Handstück siehe Abb. 3, Röntgendiffraktogramm in Abb. 4). Im Gegensatz zur massigen Erscheinungsform im Handstück, zeigt das Fragment diefür Chrysotil typische primäre Faserstruktur

verhältnismäßig unauffällig massig. Die Untersuchungen zeigten, dass untergeordnet auch Chrysotil im Serpentinit von Traföß auftritt. Interessanterweise erscheint auch die chrysotilreiche Probe $19 \mathrm{im}$ Aufschluss bzw. Handstück massig. Dies entspricht der diesbezüglichen Mitteilung in den vom deutschen Bundesministerium für Arbeit und Soziales herausgegebenen Technischen Regeln für Gefahrstoffe (TRGS, hier Nr. 517, "Tätigkeiten mit potenziell asbesthaltigen mineralischen Rohstoffen und daraus hergestellten Gemischen und Erzeugnissen", S. 31-32 [27]):

(6) Asbest bzw. Asbestminerale (faser- und nicht faserförmig) können im Gesteinsvorkommen in zwei verschiedenen Ausbildungen auftreten:

Asbest/Asbestminerale in Klüften,

Asbest/Asbestminerale im "kompakten" ungestörten Gestein.

(7) Die erste Form des Auftretens ist bei Steinbruchsbegehungen leicht zu erkennen. Die im Gestein selbst enthaltenen Asbestminerale können in der Regel erst durch petrographische Untersuchungen erkannt werden. Häufig "entstehen" Asbestfasern der zweitgenannten Form erst durch mechanische Beanspruchung der Gesteine (Aufbereitung) aus nicht faserförmigen Asbestmineralen.

Bei den bereits während der Tunnelvortriebsarbeiten ohne weitere analytische Hilfsmittel vom Geologen erkannten Asbestfasern handelt es sich daher vermutlich um Kluftfüllungen.

Die hohe Konzentration an asbestiformen Silikatfasern und der durchgeführte Test zeigen jedoch, dass die vorherrschende Quelle der asbestiformen Silikatfasern hier Antigorit, das dominante Mineral des Serpentinits von Traföß, sein muss. Allerdings ist in diesem Zusammenhang auch die Beobachtung von Bedeutung, dass auch Chrysotil im Sinne der zitierten Aussage der TRGS 517 im Gelände als massig erscheinendes Gestein auftreten kann.
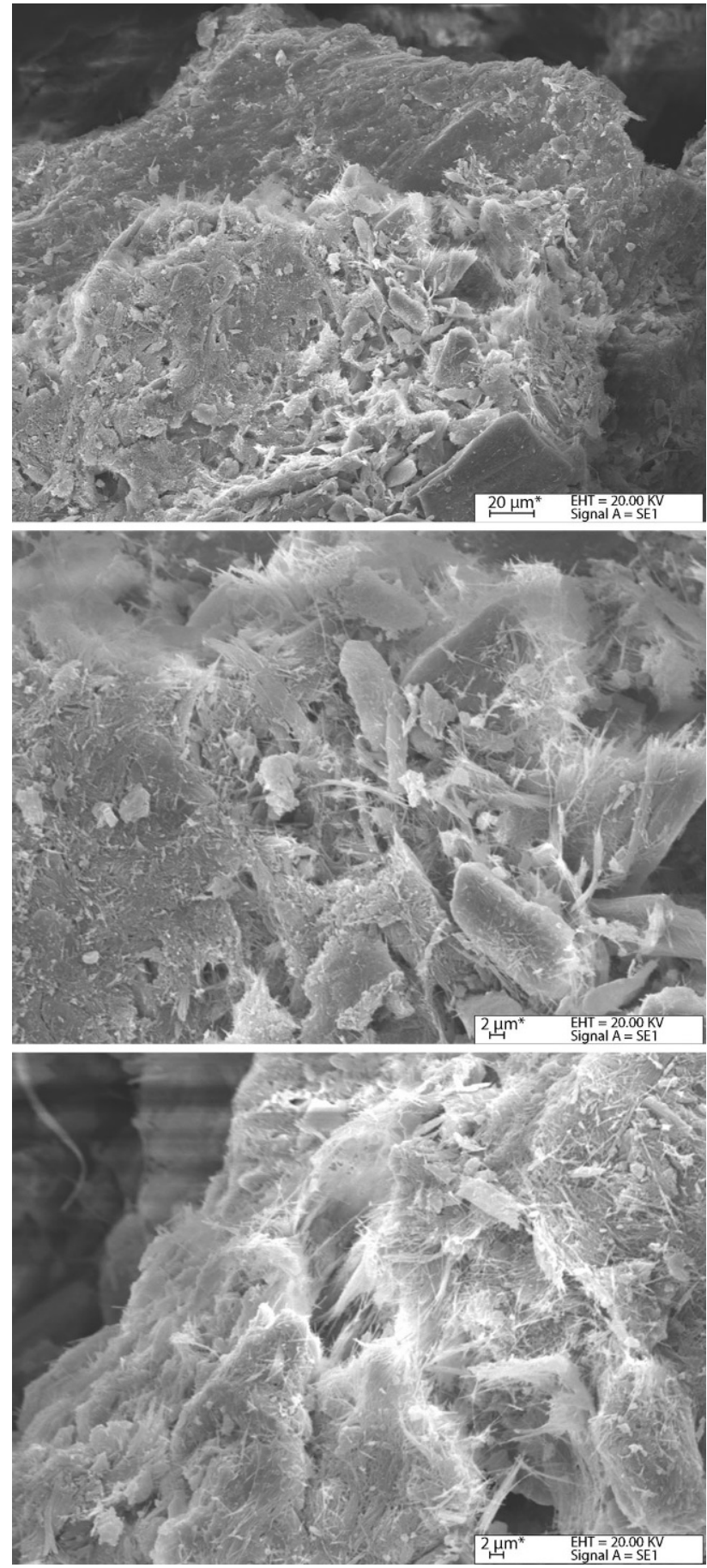

Abb. 10: Aggregate von feinkörnigem Material mitasbestiformen Fasern verschiedener Form und Größe der Probe SN2c. a Übersicht, b Detail von oben, $\mathbf{c}$ Detail eines weiteren Aggregates

\subsection{Gesundheitsschädigende Wirkung der Antigoritfasern}

\subsubsection{Vorbemerkung}

Trotz der Tatsache, dass die Gefährlichkeit von asbestiformem Antigorit in den letzten Jahren in der Literatur bereits mehrmals beschrieben wurde und in Fachkreisen bekannt 
war, ist dieser Antigorittyp erstaunlicherweise im Gegensatz zu kommerziell genutzten Asbestarten bisher nicht als Gefahrstoff klassifiziert und keinen Gesundheitsschutzregularien unterworfen, obwohl bei seiner Bearbeitung oft Faserfeinstäube entstehen und ebenso wie beim Umgang mit dem strukturell verwandten Chrysotil (siehe oben) asbesttypische Krankheitsbilder auftreten. Faseriger Antigorit wurde deshalb sogar aus Unwissenheit gelegentlich als Ersatz für Asbest verwendet, z. T. aufgrund von Verwechslung als Chrysotil vermarktet [8]. Ob Antigorit ähnlich wie Chrysotil - im Gegensatz zu Amphibolasbesten - in vivo eine relativ geringe Halbwertszeit hat, ist nicht bekannt.

Keeling et al. [7-9] untersuchten faserigen Antigorit aus einem australischen Steinbruch (Rowland Flat) und entsprechende Proben aus der Sierra Nevada (Spanien) und verglichen die Befunde mit jenen von Chrysotil aus New South Wales (Australien). Antigorit wies typische Faserbündel auf. Die genannten Autoren folgern, dass - wie sich aus den untersuchten Proben, aber auch aus polnischen und italienischen Antigorit-Vorkommen zeigt - faseriger Antigorit eine Reihe von Charakteristika wie Chrysotilasbest aufweist und somit als ein potentielles Gesundheitsrisiko einzustufen ist.

Es liegen mehrere Berichte über Erkrankungen unter Antigorit-Exponierten vor, wie sie von Asbesteinwirkungen gut bekannt sind. So wurden unter polnischen Arbeitern einer (lateritisches) Nickelerz-verarbeitenden Firma, in der eine anhaltende Belastung mit faserförmigen Antigoritstäuben bestand, 25 Asbestose-Erkrankungen diagnostiziert [13]. Dabei fand sich eine Assoziation zwischen der Antigorit-Faserzahl einerseits, den fibro- und kanzerogenen Fasern sowie dem fibro- und pathogenen Effekt des Staubes andererseits.

Entsprechende Gefährdungen werden unter Beschäftigten/Umwelt-Exponierten im Bereich der italienischen WestAlpen, wo Antigorit in Serpentiniten auftritt (Cardile et al. [11], Pugnaloni et al. [12]), sowie im Bereich des erwähnten australischen Steinbruchs angenommen (Keeling et al. [7-9]).

Baumann berichtete über erhöhte Inzidenzraten an Lungenkrebs und Mesotheliom in Neukaledonien in den letzten 30 Jahren [10]. Das Auftreten in etwa gleicher Häufigkeit in beiden Geschlechtern und oft in jungen Jahren spricht für eine Ursache in der allgemeinen Umwelt. Baumanns Untersuchungen ergaben, dass die Belastung mit antigorithaltigem Serpentinit, welcher zum Straßenbau verwendet wurde, in der Umgebung belasteter Straßen das höchste Umweltrisiko darstellte: $p<0,001$; OR 495, $95 \%$ Cl 46,2-4679,7; multivariates IRR 13;, $95 \% \mathrm{Cl} 10,2-16,6$ (OR: odds ratio, Cl: confidence interval, IRR: incidence rate ratio). In den Gebieten mit dem höchsten Erkrankungsrisiko befanden sich zahlreiche Serpentinit-Steinbrüche, in denen Antigorit das vorherrschende faserförmige Mineral darstellt. Für das Mesotheliom fand sich eine engere Assoziation mit Serpentiniten, speziell Antigorit, als für Amphibole.

\subsubsection{Tierversuche}

Wozniak et al. [13] applizierten faserförmigen Antigorit, der aus Nickel-haltigem Erz gewonnen wurde, Ratten intraperi- toneal. $80 \%$ der Tiere entwickelten Tumoren; in $77 \%$ handelte es sich um Mesotheliome. Krokydolith wies vergleichbare Ergebnisse auf, während Chrysotil wesentlich seltener Tumoren erzeugte. Es fand sich eine Korrelation zwischen der Faserzahl und dem Hydroxyprolin-Gehalt der Lunge als fibrogenem Indikator ( $r=0,96$; für Fasern mit einer Länge von mehr als $5 \mu \mathrm{m} \mathrm{r}=0,83$ ). Außerdem entwickelten die Tiere Mesotheliome. Insgesamt erwies sich Antigorit hinsichtlich der fibrogenen, karzinogenen und mutagenen Wirkung ähnlich potent wie Krokydolith [13, 14].

\subsubsection{In vitro-Untersuchungen}

Cardile et al. setzten mesenthelialen Zelllinien (MeT5-A) und Monozyten-Makrophagen (J774)-Zelllinien faserförmigem Antigorit aus [11]. Es ergab sich eine dosisabhängige Zelltoxizität, außerdem die Stimulation der Produktion von reaktiven Sauerstoffspezies, Nitrit und Prostaglandin $\mathrm{E}_{2}\left(\mathrm{PGE}_{2}\right)$. Diese Befunde belegen die Stimulation der Cyclooxygenase und die Bildung von Hydroxy- und StickoxidRadikalen. Sie stellen - wie die Autoren schlussfolgern frühe zelluläre Veränderungen hinsichtlich pathologischer und neoplastischer Entwicklungen dar. Entsprechende Untersuchungen mit einer humanen Alveolarepithel-Zelllinie (A549) ergaben eine irreguläre Verteilung von Aktin-Filamenten als Zeichen einer zellulären Toxizität [12]

\section{Schlussfolgerungen und Empfehlungen}

Die fibrogene und kanzerogene Potenz aller Asbestarten und die Auslösung der damit verbundenen Krankheitsbilder - Mesotheliom, Lungenkrebs, Larynxkrebs, Eierstockkrebs, Asbestose, Pleuraplaques/-Fibrose - ist unstrittig (Baur et al. [28], International Agency for Research on Cancer [29], WHO; [30-32]). Dabei ergeben sich lediglich gewisse Abweichungen in den Dosis-Wirkungsbeziehungen, aber keine prinzipiellen Unterschiede. Wesentliche gemeinsame pathogenetische Grundprinzipien sind dabei die durch die Faserstruktur bedingte Zytotoxizität, Fibrogenität und Bildung proinflammatorischer Zytokine und reaktiver, die DNA schädigender Radikale (National Institute of Occupational Safety and Health [33], Churg et al. [34], Mossman und Churg [35]). Alle diese Wirkungen sind, wie dargestellt, auch für Antigorit belegt. An einem entsprechenden gesundheitsgefährdenden Potenzial des vor allem in den weltweit auftretenden Serpentiniten, aber gelegentlich auch in industriell gefertigten Produkten (als Asbestersatz z. T. eingesetzt) vorkommenden, dem Weißasbest (Chrysotil) strukturell und chemisch ähnlichen Serpentinminerals Antigorit besteht kein Zweifel. Dies gilt sowohl für primär faserigen (asbestiformen) Antigorit, wie auch für asbestiformen Antigorit, dessen Fasern durch mechanische Bearbeitung des als Gestein massig erscheinenden antigoritischen Serpentinits gebildet werden. Belegt wird die gesundheitsgefährdende Wirkung von asbestiformem Antigorit sowohl durch die dargelegten tierexperimentellen Untersuchungen, als auch durch klinische Daten exponierter Personen. Eine entsprechende Warnung sprechen auch Fitzgerald und Harty [36] und Baumann et al. 
[37] aus. Dabei ist insbesondere auf die Beobachtung von Mesotheliomen und Asbestose hinzuweisen. Außerdem ist von Bedeutung, dass Antigorit nicht selten gleichzeitig mit weiteren Asbestmineralen vorkommt und dadurch im Einzelfall die Abgrenzung der pathogenen Bedeutung der nebeneinander gleichzeitig auftretenden faserförmigen Staubkomponenten schwierig bzw. nicht möglich ist (vgl. Anmerkungen von Fitzgerald und Harty, [36]).

Aus Vorgenanntem leitet sich ab, dass die Exposition gegenüber antigorithaltigen Feinstäuben grundsätzlich zu vermeiden ist und gleichartige Umweltschutz- und Arbeitsschutzregularien bis hin zum Anwendungsverbot wie für andere Asbestarten angezeigt sind.

Die hier veröffentlichten Untersuchungsergebnisse, wie auch die internationale Literatur, zeigen somit, dass auch bei antigoritischen Serpentiniten grundsätzlich mit einem Gesundheitsrisiko bei Tunnelarbeiten und ähnlichen Tätigkeiten zu rechnen ist. Wichtig ist in diesem Zusammenhang, dass nach Normmethoden (ÖNORM M 9405, Messung von Asbestfaserkonzentrationen in der Luft [15]) Chrysotil und fibröser Antigorit nicht unterschieden werden können.

Bei Durchörterung oder sonstiger massiver Bearbeitung von Serpentiniten sollte daher unbedingt von Anfang an mit entsprechenden Messungen des Fasergehaltes der Umgebungsluft und, bei geringstem Verdacht, mit Sicherheitsmaßnahmen begonnen werden. Dissauer et al. beschreiben entsprechende Arbeitsschutzmaßnahmen [1]. Dabei ist zu berücksichtigen, dass Serpentinite, wie jener von Traföß/ Steiermark, in Orogenen des alpinen Typs weit verbreitet sind.

Abschließend wird es aus fachlicher Sicht für notwendig gehalten, faserigen bzw. bei Bearbeitung zur Faserbildung neigenden Antigorit als asbestiformen Antigorit, analog zum Chrysotil, in die Gruppe der Asbestminerale aufzunehmen, mit den entsprechenden rechtlichen und präventiven Konsequenzen.

Funding. Open access funding provided by Montanuniversität Leoben.

Open Access Dieser Artikel wird unter der Creative Commons Namensnennung 4.0 International Lizenz (http://creativecommons.org/licenses/ by/4.0/deed.de) veröffentlicht, welche die Nutzung, Vervielfältigung, Bearbeitung, Verbreitung und Wiedergabe in jeglichem Medium und Format erlaubt, sofern Sie den/die ursprünglichen Autor(en) und die Quelle ordnungsgemäß nennen, einen Link zur Creative Commons Lizenz beifügen und angeben, ob Änderungen vorgenommen wurden.

\section{Literatur}

1. Dissauer, J.; Präsent, G.; Schwab, P. Bruck expressway S35 contract 22 - Tunnelling through serpentinite - Brucker Schnellstraße S35 Baulos 22 - Vortriebe im Serpentinit. Geomechanik und Tunnelbau/ Geomechanics and Tunnelling, 2 (2009), no.5, pp 483-493

2. Neubauer, F: Bau und Entwicklungsgeschichte des Rennfeld-Mugelund des Gleinalm-Kristallins (Ostalpen). Abhandlungen der Geologischen Bundesanstalt, Bd. 42 (1988)

3. Thalhammer, O. R.; Ebner, F.; Horkel, K.; Mali, H.: Der UltramafitKomplex von Kraubath (The Kraubath Ultramafic Massif). Journal of Alpine Geology, 53 (2010), S. 137-158

4. Vortisch, W.; Baur, X.: Asbestiform Antigorite: A dangerous mineral in Serpentinites. A plea to treat asbestiform antigorite as an asbestos group mineral in terms of its occupational health safety effects. Neues Jahrbuch für Mineralogie, 195 (2018), H. 1 (im Druck), appeared online 16.11.2017 (https://doi.org/10.1127/njma/2017/0070)
5. Fitz Gerald, J. D.; Eggleton, R. A.; Keeling, J. L.: Antigorite from Rowland Flat, South Australia: asbestiform character. European Journal of Mineralogy, 22 (2010), pp 525-533

6. Groppo, C.: Compagnoni, R.: Ubiquitous fibrous antigorite veins from the Lanzo Ultramafic Massif, Internal Western Alps (Italy): characterization and genetic conditions. Periodico di Mineralogia, 76 (2007), pp 169-181

7. Keeling, J.; Raven, M. D.; McClure, S. G.: Identification of fibrous mineral from Rowland Flat area, Barossa Valley, South Australia. Department of Primary Industries and Resources: Report Book, 2006/2

8. Keeling, J.; Raven, M. D.; Self, P. G.; Eggleton, R. A.: Asbestiform Antigorite Occurrence in South Australia. Ninth International Congress for Applied Mineralogy, Brisbane, QLD, 8-10 September 2008, pp 329-336

9. Keeling, J.; Raven, M. D.; Self, P. G.: Asbestiform antigorite - implications for the risk assessment of fibrous silicates. 21st Australian Clay Minerals Conference - Brisbane, August 2010, Extended Abstracts

10. Baumann, F: Environmental exposure to carcinogenic fibers due to serpentinite paved roads in New Caledonia; Lessons in environmental epidemiology and in prevention. Geological Society of America, Cordilleran Section, 108th Annual Meeting, Abstracts with Programs, 44 (2012), no.3, p. 8

11. Cardile, V.; Lombardo, L.; Belluso, E.; Panico, A.; Capella, S.; Balazy, M.: Toxicity and carcinogenicity mechanisms of fibrous antigorite. International Journal of Environmental Research and Public Health, 4 (2007), iss. 1, pp 1-9

12. Pugnaloni, A.; Giantomassi, F; Lucarini, G.; Capella, S.; Mattioli Belmonte, M.; Orciania, M.; Belluso, E.: Effects of asbestiform antigorite on human alveolar epithelial A549 cells: A morphological and immunohistochemical study. Acta Histochimica, 112 (2010), pp 133-146

13. Wozniak, H.; Wiecek, E.; Stetkiewicz, J.: Fibrogenic and carcinogenic effects of antigorite. Polish Journal of Occupational Medicine, 1 (1988), nr. 3, pp 192-202

14. Wozniak, H.; Wiecek, E.; Stetkiewicz, J.; Wyszynska, K.: Experimental carcinogenicity and mutagenicity of non-asbestos natural fibres - preliminary report. Polish Journal of Occupational Medicine and Environmental Health, 6 (1993), pp 55-56

15. ÖNORM M 9405 (01.10.1993): Messung von Asbestfaserkonzentrationen in der Luft

16. Baur, X., Woitowitz, H. J., Budnik, L. T., Egilman, D., Oliver, C., Frank, A., Soskolne, C. L., Landrigan, P. J. \& Lemen, R. A.: Asbestos, asbestosis, and cancer: The Helsinki criteria for diagnosis and attribution. Critical need for revision of the 2014 update. American Journal of Industrial Medicine, 60 (2017), no. 5, pp 411-421

17. Egilman, D.; Bird, T.: Short fiber tremolite free chrysotile mesothelioma cohort revealed. American Journal of Industrial Medicine, 59 (2016), no. 3, pp 196-199

18. Lemen, R. A.: Asbestos in brakes: exposure and risk of disease. American Journal of Industrial Medicine, 45 (2004), no. 3, pp 229-237

19. VDI 3492:2013-06: Messen von Innenraumluftverunreinigungen; Messen von Immissionen; Messen anorganischer faserförmiger Partikel; Rasterelektronenmikroskopisches Verfahren. Berlin: Beuth, 2013

20. Bailey, S. W.: Structures of layer silicates, in: Brindley, G. W.; Brown, G. (eds.): Crystal structures of clay minerals and their $x$-ray identification. London: Mineralogical Society, Monograph No. 5, 1984

21. Wicks, F. J.: O'Hanley, D. S.: Serpentine minerals: Structures and petrology, in: Bailey, S. W. (ed.): Hydrous phyllosilicates (exclusive of micas), Reviews in Mineralogy, 19 (1988), pp 91-167

22. Dódony, I.; Busek, P. R.: Serpentines close-up and intimate: an HR TEM view. International Geology Review, 46 (2004), pp 507-527

23. Niedermayr, G.; Bauer, C.; Bernhard, F.; Blass, G.; Bojar, H.-P.; Brandstätter, F.; Gröbner, J.; Hammer, V. M. F.; Koch, G.; Kolitsch, U.; Leikauf, B.; Loránth, C.; Poeverlein, W.; Postl, W.; Prasnik, H.; Schachinger, T.; Tomazic, P; Walter, F.: Neue Mineralfunde aus Österreich LVII. Carinthia II, 198/118 (2008), S. 223-274

24. Siivola, J.; Schmid, R.: List of Mineral Abbreviations, in: Recommendations by the IUGS Subcommission on the Systematics of Metamorphic Rocks, Internetversion 01.02.2007. Zugegriffen: 15.11.2017 
25. Anthony, J. W.; Bideaux, R. A.; Bladh, K. W.; Nichols, M. C. (eds.): Handbook of Mineralogy, Chantilly, VA: Mineralogical Society of America, 2001, http://www.handbookofmineralogy.org/. Zugegriffen: 15.11.2017

26. Newman, A. C. D.; Brown, G.: The chemical constitution of clays, in: Newman, A. C. D. (ed.): Chemistry of clays and clay minerals. London: Mineralogical Society Monograph, No 6 (1987)

27. Technische Regeln für Gefahrstoffe (TRGS) 517: Tätigkeiten mit potenziell asbesthaltigen mineralischen Rohstoffen und daraus hergestellten Gemischen und Erzeugnissen. Ausgabe Februar 2013, Bekanntgabe durch Bundesministerium für Arbeit und Soziales, 2013

28. Baur, X.; Schneider, J.; Woitowitz, H. J.; Velasco Garrido, M.: Do advers health effects of chrysotile and amphibole asbestos differ? Pneumologie, 66 (2012), Ausg. 8, S. 497-506

29. International Agency for Research on Cancer: Asbestos (Chrysotile, Amosite, Crocidolite, Tremolite, Actinolite and Anthophyllite), in: IARC Monographs: A Review of Human Carcinogens: Arsenic, Metals, Fibres, and Dusts, $100 \mathrm{C}$ (2012), pp 219-309, http://monographs. iarc.fr/ENG/Monographs/vol100C/

30. WHO (1998): Environmental Health Criteria, 203. Chrysotile asbestos, http://www.inchem.org/documents/ehc/ehc/ehc203.htm. Zugegriffen: 15.11.2017
31. WHO (2017): Asbestos: Elimination of asbestos related diseases, WHO Fact Sheet N³43, http://www.who.int/mediacentre/factsheets/ fs343/en/. Zugegriffen: 15.11.2017

32. WHO (2014): Chrysotile asbestos, http://www.who.int/ipcs/ assessment/public_health/chrysotile_asbestos_summary.pdf. Zugegriffen: März 2017

33. National Institute of Occupational Safety and Health: Asbestos fibers and other elongate mineral particles: state of the science and roadmap for research. Current Intelligence Bulletin 62. Department of Health and Human Services, Centers for Disease Control and Prevention, DHHS (NIOSH) Publication Number 159 (2011)

34. Churg, A.; Wright, J.; Gilks, B.; Dai, J.: Pathogenesis of Fibrosis Produced by Asbestos and Man-Made Mineral Fibers: What Makes a Fiber Fibrogenic? Inhalation Toxicology, 12 (2000), Suppl. 3, pp 15-26

35. Mossman, B. T.; Churg, A.: Mechanisms in the Pathogenesis of Asbestosis and Silicosis. American Journal of Respiratory and Critical Care Medicine, 157 (1998), no. 5, pp 1666-1680

36. Fitzgerald, S. M.; Harty, E. A.: Antigorite: Is It the Forgotten Asbestos? Professional Safety, 59 (2014), no. 08, pp 43-48

37. Baumann, F.; Ambrosi, J.-P.; Carbone, M.: Asbestos is not just asbestos: an unrecognised health hazard. The Lancet Oncology, 14 (2013), no. 7, pp 576-578 